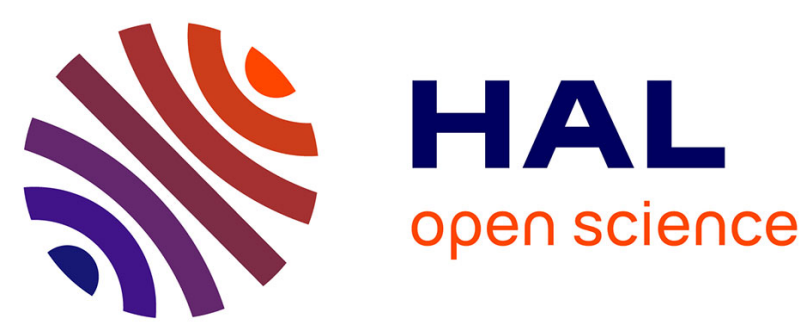

\title{
Multiphoton $\mathrm{k}$-resolved photoemission from gold surface states with 800-nm femtosecond laser pulses
}

Fausto Sirotti, Nathan Beaulieu, Azzedine Bendounan, Mathieu G. Silly,

Christian Chauvet, Grégory Malinowski, Guido Fratesi, Valérie Véniard, Giovanni Onida

\section{To cite this version:}

Fausto Sirotti, Nathan Beaulieu, Azzedine Bendounan, Mathieu G. Silly, Christian Chauvet, et al.. Multiphoton k -resolved photoemission from gold surface states with 800-nm femtosecond laser pulses. Physical Review B: Condensed Matter and Materials Physics (1998-2015), 2014, 90 (3), pp.35401 35401. 10.1103/PhysRevB.90.035401 . hal-01381241

\section{HAL Id: hal-01381241 \\ https://hal.univ-lorraine.fr/hal-01381241}

Submitted on 14 Oct 2016

HAL is a multi-disciplinary open access archive for the deposit and dissemination of scientific research documents, whether they are published or not. The documents may come from teaching and research institutions in France or abroad, or from public or private research centers.
L'archive ouverte pluridisciplinaire HAL, est destinée au dépôt et à la diffusion de documents scientifiques de niveau recherche, publiés ou non, émanant des établissements d'enseignement et de recherche français ou étrangers, des laboratoires publics ou privés. 


\title{
Multiphoton $\boldsymbol{k}$-resolved photoemission from gold surface states with 800-nm femtosecond laser pulses
}

\author{
Fausto Sirotti, ${ }^{1,{ }^{*}}$ Nathan Beaulieu, ${ }^{1}$ Azzedine Bendounan, ${ }^{1}$ Mathieu G. Silly, ${ }^{1}$ Christian Chauvet,,${ }^{1}$ Gregory Malinowski, ${ }^{2, \dagger}$ \\ Guido Fratesi, ${ }^{3,4,5}$ Valérie Véniard, 5,6 and Giovanni Onida ${ }^{3,5}$ \\ ${ }^{1}$ Synchrotron SOLEIL, L'Orme des Merisiers, Saint-Aubin, 91192 Gif-sur-Yvette, France \\ ${ }^{2}$ Laboratoire de Physique des Solides, Université Paris-Sud, CNRS UMR 8502 \\ ${ }^{3}$ Dipartimento di Fisica, Università degli Studi di Milano, Milano, Italy \\ ${ }^{4}$ CNISM and Dipartimento di Scienza dei Materiali, Università di Milano-Bicocca, Via Cozzi 53, 20125 Milano, Italy \\ ${ }^{5}$ European Theoretical Spectroscopy Facility (ETSF) \\ ${ }^{6}$ Laboratoire des Solides Irradiés, École Polytechnique, CNRS-CEA/DSM, 91128 Palaiseau, France
}

(Received 20 August 2013; revised manuscript received 12 June 2014; published 2 July 2014)

\begin{abstract}
We measure direct multiphoton photoemission of the $\mathrm{Au}(111)$ surface state with 800 -nm laser pulses. We observe the parabolic dispersion in the angular distribution of photoelectrons having absorbed between four and seven photons. The $\mathbf{k}_{\|}$dispersion we measure can be explained in terms of Shockley-state replicas, with a nascent hot electrons distribution at $\mathbf{k}_{\|}$above the Fermi level. Moderate laser power densities, of the order of $100 \mathrm{GW} / \mathrm{cm}^{2}$, resulted in large electron yields, indicating the importance of multiphoton excitations to define the electronic and magnetic properties of matter in the first hundred femtoseconds after laser excitation.
\end{abstract}

DOI: 10.1103/PhysRevB.90.035401

PACS number(s): 79.20.Ws, 73.20.-r, 79.60.-i

\section{INTRODUCTION}

Laser-induced multiphoton photoemission (PE) from metal surfaces has been known since the seventies [1]. While above-threshold PE has been observed for different materials and sample shapes [2-9], studies of k-resolved multiphoton PE from flat surfaces are still scarce. Recently, two- and three-photon PE spectra have been measured in angle-resolved experiments on $\operatorname{Ag}(111)$ [10] and $\mathrm{Cu}(110)$ [11] using a relatively low laser intensity, corresponding to a power density of the order of $10 \mathrm{GW} / \mathrm{cm}^{2}$ at a wavelength of $\lambda=400 \mathrm{~nm}$. Higher power densities, up to about one order of magnitude, are still below the surface damage threshold and can be used to probe solid surfaces by creating strong nonequilibrium electronic distributions, with subsequent lattice-mediated equilibrium taking place only in the picosecond range. Such ultrashort pulses ( $\leqslant 50 \mathrm{fs}$ ) can depose an energy of a few millijoules per square centimeter, corresponding to a power density of the order of $100 \mathrm{GW} / \mathrm{cm}^{2}$, without inducing structural modifications. New phenomena can be observed at time scales below $\simeq 100 \mathrm{fs}$, making ultrafast intense laser pulses an experimental tool of growing importance in several fields. Different regimes of laser-induced emission exist. In terms of intensity, a transition from the multiphoton regime towards tunneling (optical-field emission) has been well known since the sixties [12,13]. Moreover, a pulse duration shorter than or of the same order as the electromagnetic wave period (fewcycle pulses) as in recent experiments [14] further complicates the picture by introducing a dependence on the timing of the oscillations with respect to the pulse peak [15] — known as carrier envelope phase sensitivity-and strongly increasing the intrinsic bandwidth, i.e., decreasing the energy resolution. For an 800-nm laser and a pulse duration of the order of

\footnotetext{
*fausto.sirotti@synchrotron-soleil.fr

${ }^{\dagger}$ Current address: Institut Jean Lamour, CNRS UMR 7198, Université de Lorraine, 54506 Vandoeuvre-lès-Nancy, France.
}

$50 \mathrm{fs}$, carrier envelope phase phenomena and the intrinsic loss of energy resolution can be safely neglected. In terms of peak intensity, at $\simeq 100 \mathrm{GW} / \mathrm{cm}^{2}$ tunneling phenomena are still negligible for a flat metal surface at infrared wavelengths (Keldysh parameter $\gamma>1$ [12]), so that a multiphoton regime can be assumed, at variance with the case of emission from sharp objects like nanotips, where a strong field enhancement is induced by the small curvature $[8,9,16,17]$. For instance, ultrafast demagnetization [18] and optical manipulation of magnetic order experiments [19-21], of growing importance in the last decade, are well within the multiphoton regime. In this context, a lot of attention has been devoted to the role of hot electrons, generated by the light-matter interaction and subsequently transported through the sample/device, eventually leading to a loss of magnetization [22-28]. Clearly, a high density of excited electrons is expected to affect the transient electronic and magnetic response. The latter can indeed become substantially different from the equilibrium one and should be taken into account in the interpretation of the wide class of studies currently performed at such power densities.

In this work we show experimental evidence of direct angle-resolved PE processes involving a large number (from four to seven) of photons. Moreover, based on a theoretical modeling of the strong-field multiphoton absorption process in the presence of a periodically modulated potential, we estimate the number of multiexcited electrons having absorbed fewer than four photons, i.e., the electrons which, within the laser pulse, populate the bound empty states of the metal.

We perform angular resolved $\mathrm{PE}$ measurements on $\mathrm{Au}(111)$ using an 800-nm (1.55-eV) laser source. The use of this laser wavelength allows us to study multiphoton PE signals for processes involving up to seven photons, absorbed by electrons which originate only from an energy region within $1.55 \mathrm{eV}$ below the Fermi level. Namely, direct electronic transitions from $d$ bands and deep surface-projected bulk bands are excluded, at variance with the case of 400-nm photons, hence simplifying the analysis of the results. 


\section{EXPERIMENTAL AND THEORETICAL METHODS}

Experiments were performed on the UHV-Photoemission experimental station of the TEMPO beamline at the SOLEIL synchrotron radiation facility [29]. The soft X-ray beam and the 50-fs pulses FWHM from a Coherent REGA laser impinge on the same sample. The laser beam illuminates an area of about $6 \times 10^{-4} \mathrm{~cm}^{2}$ on the sample, resulting in a maximum (peak) intensity of $120 \mathrm{GW} / \mathrm{cm}^{2}$. Such a value is low enough to avoid surface heating and degradation: experiments can be performed for several hours without visible modification of the spectra. The experimental station is equipped with a twodimensional Scienta SES 2002 analyzer with an acceptance angle of $12^{\circ}$ in the horizontal plane. The measurements are performed on a well-ordered and clean $\mathrm{Au}(111)$ surface obtained after suitable sputtering and annealing cycles; all experiments are performed at liquid nitrogen temperature and a pressure of less than $10^{-10}$ mbar.

PE model calculations were performed within a onedimensional (1D) one-electron scheme similar to that used in Refs. [30] and [31]. We use a regularized atomic potential $V(x)$, defined as

$$
V(x)=\frac{V_{0}}{\sqrt{x^{2}+1}} e^{-k_{\mathrm{scr}}|x|},
$$

summed over a chain of 220 "atoms" [31]. Here $k_{\mathrm{scr}}=$ $1.98 n_{0}^{1 / 6}$ is a static screening wave vector $\left(n_{0}\right.$ being the free electron density of the metal) and $x$ is the coordinate in atomic units. Externally to the chain, we add to the potential the constant $V_{1}$, tuning $V_{0}$ and $V_{1}$ to mimic an ideal Au crystal surface ( $V_{0}=-0.25$ a.u. and $V_{1}=0.28$ a.u.).

The 1D time-dependent Schrödinger equation is then solved on a spatiotemporal grid. The initial state is chosen to be a surface state and is propagated in time. The interaction between the electron and the electric field is described in the velocity gauge, $H_{\mathrm{int}}=-\frac{1}{c} \mathbf{A}(t) \cdot \mathbf{p}$, and the electric field is taken, in the dipole approximation, as $E(t)=E_{0} f(t) \sin \omega t$, where $f(t)$ denotes the pulse envelope linearly ramped on and off during one laser period. It is related to the vector potential through the relation $E(t)=-\frac{1}{c} \frac{d \mathbf{A}}{d t}$. The time evolution of the wave function is obtained using the split operator method [32]. The convergence of the numerical scheme needs 30000 time steps per optical period. In order to avoid reflections of the electronic density from the boundaries of the box, we used an absorber.

To simulate the PE yield, we propagate the inital wave function $\Psi$ until the end of the pulse $\left(t=T_{\text {pulse }}\right)$. At this point, the spectrum is constructed through a standard spectral analysis [33],

$$
P_{n}(E, \Delta E)=\left\langle\Psi\left(T_{\text {pulse }}\right)\left|W_{n}\right| \Psi\left(T_{\text {pulse }}\right)\right\rangle,
$$

where $W_{n}$ is a window operator defined by

$$
W_{n}(E, \Delta E)=\frac{\Delta E^{2 n}}{\left(H_{0}-E\right)^{2 n}+\Delta E^{2 n}}
$$

and $H_{0}$ is the field-free Hamiltonian. The integer $n$ was set to 2 in the present analysis.

A reference $\mathrm{Au}(111)-(1 \times 1)$ band-structure calculation for a 99-layer-thick $\mathrm{Au}$ slab, used for the analysis of Brillouin-zone (BZ) folding below, was performed within standard density functional theory, using the Perdew-Burke-
Ernzerhof approximation for the exchange and correlation functional [34], with pseudopotentials and plane waves [35].

\section{RESULTS AND DISCUSSION}

A reference one-photon PE spectrum, taken with a $60-\mathrm{eV}$ synchrotron radiation source, displays the well-known (Rashba split) parabolic surface state [36], as shown in the top panel in Fig. 1, where the resulting PE intensity map is reported. The corresponding electron effective mass $m^{*}=$ $0.26 m_{e}$ and the distance of the minimum from the Fermi level of $0.47 \mathrm{eV}$, used to draw the dotted lines, match well the literature data [36].

When the excitation source is switched to the 800-nm laser, we obtain the spectra shown in Fig. 1, where the color intensity is presented in logarithmic scale. The final-state energy refers to the Fermi energy, and the emission angle $\left( \pm 5^{\circ}\right)$ spans a $\mathbf{k}_{\|}$ region which, for a final energy of $5 \mathrm{eV}(10 \mathrm{eV})$, corresponds to $0.1(0.14) \AA^{-1}$. Four structures are observed, regularly spaced in energy. Dotted lines indicate the (Rashba split) angular dispersions for the same effective mass, $m^{*}=0.26 m_{e}$, as that of the Shockley state. The first three structures are clearly visible in Fig. 1, while the fourth one, at about $10.3 \mathrm{eV}$, can be better seen by plotting the integrated PE intensities over a $1^{\circ}$ range around normal emission (see circles in Fig. 2; discussed below in more detail). While the lower-final-state-energy side of the periodic structures in Fig. 1 appear to follow well the parabolic dispersion of the Shockley state corresponding to absorption of four, five, six, and seven photons of $800 \mathrm{~nm}$, as depicted in the left panel in Fig. 1, the upper edge is flat
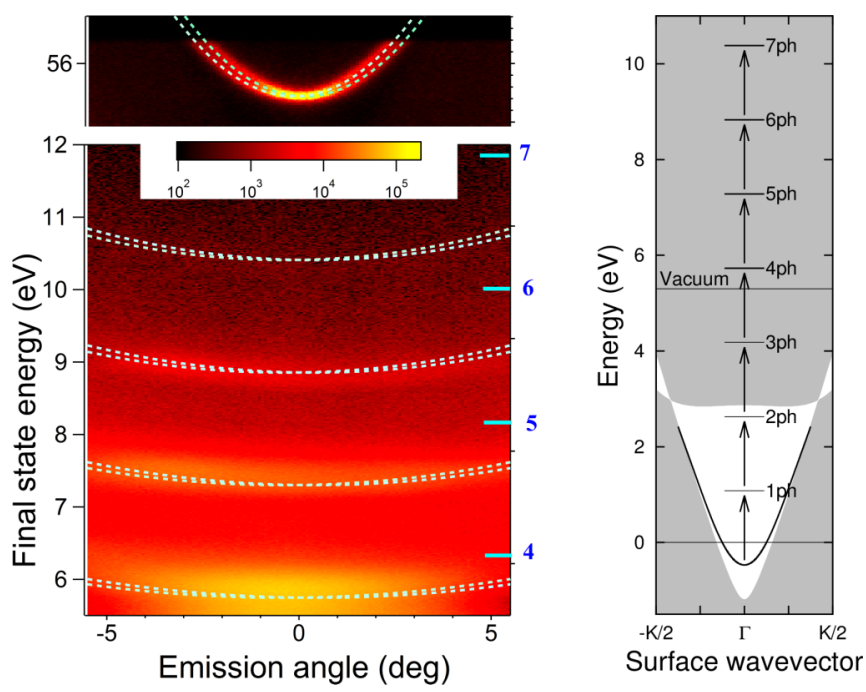

FIG. 1. (Color online) Left: Angular resolved photoelectron spectroscopy of the $\mathrm{Au}(111)$ surface state measured around normal emission with $60 \mathrm{eV}$ synchrotron radiation (top) and 800-nm femtosecond laser pulses (bottom). Dotted lines represent the parabolic angular dispersion of the Rashba split surface states with $m^{*}=0.26 m_{e}$ and the relative final-state energy. Final-state energies corresponding to the absorption of four, five, six, and seven $800-\mathrm{nm}$ photons from the Fermi level are shown by the horizontal ticks on the right axis. Right: Schematic processes for multiphoton absorption from the surface state (solid line) of $\mathrm{Au}(111)$. The shaded area represents surface-projected bulk states. 


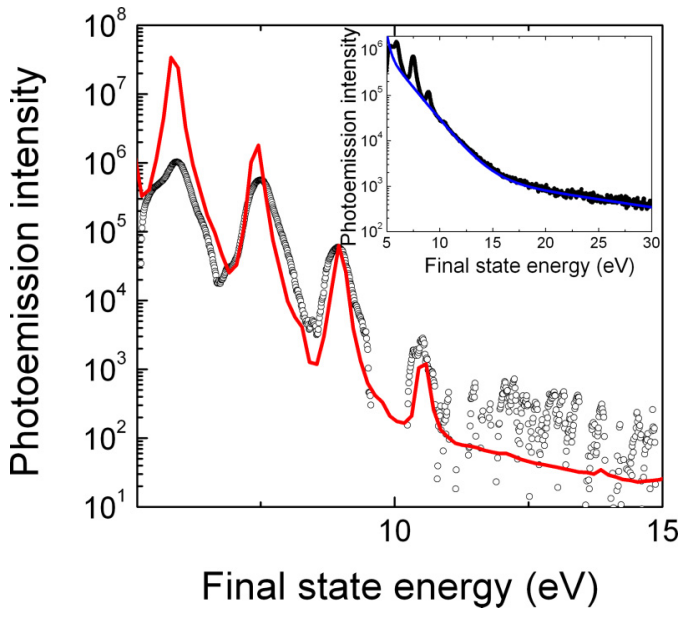

FIG. 2. (Color online) Primary photoemission intensity obtained experimentally within $1^{\circ}$ of the $\Gamma$ direction (open circles), after subtracting the background. The solid (red) curve is the model theoretical prediction (see text). Inset: Original data and subtracted background.

on the Fermi-level side of the photoelectron distribution. The observation of features closely related to the surface state of $\mathrm{Au}(111)$ further corroborates the absence of degradation and that the surface roughness does not increase significantly under experimental conditions.

Due to the low photon energy we use, at normal emission only the Shockley-state dispersion near $\Gamma$ is accessible. Regions at higher $\mathbf{k}_{\|}$values can be explored only after rotating the sample normal out of the analyzer axis. The ARPES measured at $5^{\circ}$ off normal emission and an incoming photon flux reduced to $50 \%$ is presented in the left panel in Fig. 3, focusing on the energy region around the five-photon signal. The dashed lines

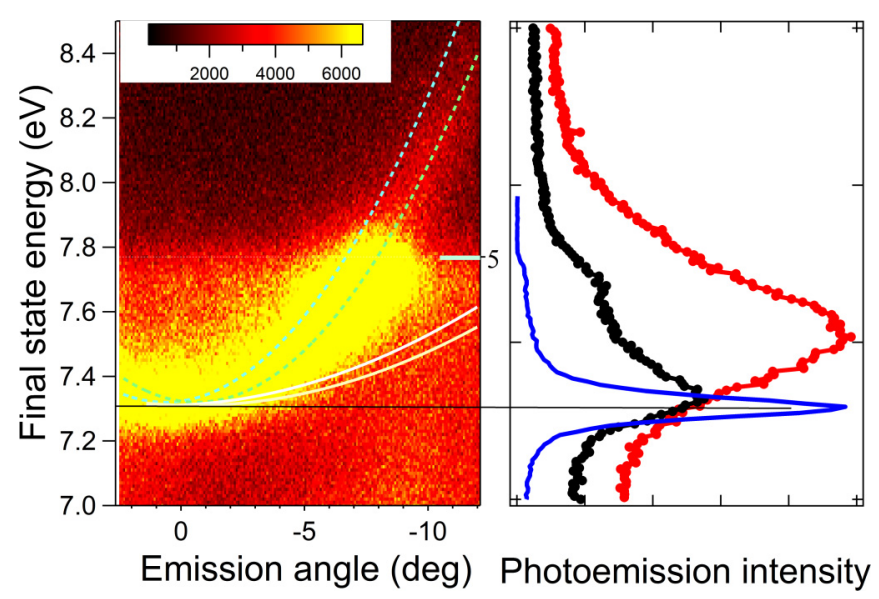

FIG. 3. (Color online) Left: Angular resolved photoelectron spectroscopy of the $\mathrm{Au}(111)$ surface state measured rotating the sample normal by $5^{\circ}$ away from the analyzer axis. The dashed and solid lines represent the parabolic angular dispersion of the Rashba split surface states with $m^{*}=0.26 m_{e}$ and $m^{*}=1 m_{e}$, respectively. Right: Photoemission spectra integrated over $1^{\circ}$ around $\Gamma$ measured for $120 \mathrm{GW} / \mathrm{cm}^{2}$ [rightmost (red) curve] and $60 \mathrm{GW} / \mathrm{cm}^{2}$ (black curve). The leftmost, thin solid (blue) curve represents the symmetric line shape measured at $60 \mathrm{eV}$ photon energy. reported in the angular distribution refer to the Shockley state, as in Fig. 1 (as a comparison, parabolas with $m^{*}=m_{e}$ are also drawn). The intersection of the Shockley state with the Fermi level is clearly visible; it is indicated by the reduction of PE intensity at larger angles. The main measured structures can be interpreted in terms of electrons from the surface state having conserved their $\mathbf{k}_{\|}$crystal momentum during the multiphoton excitation. A closer look at the five-photon region reveals a partial "filling" of the parabola up to the Fermi level, with an increased intensity at $\Gamma$ and a nondispersive upper edge. This can be seen more clearly in the right panel in Fig. 3, which shows the intensity of the five-photon peak integrated over a $1^{\circ}$ emission angle around $\Gamma$. The rightmost (red) curve corresponds to a laser power density of $120 \mathrm{GW} / \mathrm{cm}^{2}$, while the black one was obtained from the $50 \%$ reduced power: in the latter case a high-energy shoulder, corresponding to electrons photoemitted from the Fermi level, is clearly visible. We also report the line shape measured with $60 \mathrm{eV}$ photon energy as a reference [thin, leftmost (blue) curve]. Possible explanations of this effect include incoherent processes, such as scattering with defects or indirect (phonon-assisted) transitions.

Finally, we also see in Fig. 3 the appearance of photoemitted electrons coming from states with $k_{\|}$larger than the Fermi wave vector $k_{\mathrm{F}}$, showing the population of the Shockley state also beyond the Fermi energy during the 50-fs laser excitation. These electrons, initially excited, are incoherently decaying through a sequence of downward transitions, resulting in the final population of the (longer-lived) excited states immediately above $k_{\mathrm{F}}$. The 31-fs [36] lifetime estimated for the $\mathrm{Au}(111)$ surface state well matches the laser pulse duration to justify this observation. A similar effect is also observed in the six-photon signal, where it shows up at a smaller emission angle, in agreement with the higher final-state energy.

A further feature, more evident in the inset in Fig. 2, is a structureless background superimposed on the well-defined surface state replicas. It extends to a much higher kinetic energy (about $50 \mathrm{eV}$ ) and is attributed to electrons accelerated by the local space charge created by the laser pulse. Their contribution to the measured energy distribution curve is a broad background which can be well reproduced with three exponential decay functions. The energy decay lengths are $0.22,1.6$, and $12 \mathrm{eV}$, to cover the whole kinetic energy range. The shortest decay length is independent on the laser power density and can be attributed to the secondary electrons created in the solid, in analogy with normal PE experiments. The two longer decay lengths are chosen to interpolate well the whole energy dependence of the measured background at a given laser power density, and for the present discussion their role is limited to facilitating the extraction of the contribution from the multiphoton ARPES process.

When the $\mathrm{Au}(111)$ band structure is considered (see right panel in Fig. 1), the only occupied states available within a binding energy region of $1.55 \mathrm{eV}$ (accessible with our low photon energy) are the parabolic surface states whose signature is clearly seen in the spectra discussed above. The $d$ bands observed in noble metal PE with 400-nm laser pulses are, for normal emission, deep enough to be excluded from the PE process. Moreover, the bottom of the bulk empty bands at $\Gamma$ is found at about $3 \mathrm{eV}$ above the Fermi level. Image states exist but are less important than in $\mathrm{Ag}$, due to their overlapping 
with continuous bulk bands [37]. Hence, in a perfect $\mathrm{Au}(111)$ $(1 \times 1)$ surface, no states are available at $\Gamma$ in the interval -1 to $3 \mathrm{eV}$ with respect to the Fermi level.

The emission of electrons which have absorbed more photons than the minimum number needed to overcome the surface barrier is usually termed above-threshold PE (ATP), in analogy with the process of above-threshold ionization in atoms. However, there are important differences in solids with respect to the atomic case: the ionization potential is a well-defined intrinsic property of the atom, so that bound and unbound electrons can be defined and clearly behave differently. Instead, electrons in a solid can be excited from an occupied band into an empty one, continue to propagate through the periodic potential of the solid (no matter the value of the work function), and possibly absorb further photons, still being able to exchange momentum with the crystal lattice. It is only in the presence of a surface that the concepts of work function and vacuum energy come into play, distinguishing between those electrons which will continue to propagate away in the vacuum and those which will not. It is hence important to describe the multiphoton PE process by taking into account the periodic nature of the crystal potential. Recent work in this sense has been performed by several authors [38,39].

In order to estimate the photoemitted intensities, the use of a one-electron framework is justified since the laser frequency is much lower than the plasma frequency of the valence electron gas. The field intensity, of the order of $0.1 \mathrm{~V} / \AA$, is much lower than the critical field at which the metallic screening and band structure properties would change towards those of a semimetal [40]. Similarly, ponderomotive shifts, which are relevant in nanotip experiments at similar laser fluencies because of the strong field enhancement induced by the small curvature $[8,9,17]$, are negligible in our case: roughness effects are small on a flat and well-ordered surface [41], and without curvature-induced field enhancement the value of the ponderomotive shift is less than $10 \mathrm{meV}$ at $120 \mathrm{GW} / \mathrm{cm}^{2}$ and an 800-nm wavelength [42]. We follow the approach in Refs. [30] and [31], where the electrons are assumed to move in the static background field of the ions forming the lattice. The lattice is approximated by a periodic linear and finite chain of ions, with potential given by Eq. (1). The corresponding field-free eigenvalues are reported in Fig. 4, while the bottom panel in the inset presents a sketch of the potential. The upper panel in the same inset shows the wave function corresponding to one of the two surface states lying inside the gap and clearly localized at the surface.

This state lies $5.8 \mathrm{eV}$ below the vacuum, which mimics the experimental situation of $\mathrm{Au}$ surface states at the $\Gamma$ point described earlier. However, this potential also introduces intermediate states accessible within a one-photon absorption process from the surface state [not present in the band structure of perfect $\mathrm{Au}(111)]$, which are discussed below.

PE starting from one of these two degenerate states was modeled by solving the time-dependent Schrödinger equation on a spatiotemporal grid $[43,44]$. Note that due to our 1D model, we are restricted to an electric field perpendicular to the gold surface, which is, nevertheless, compatible with the experiments performed at grazing incidence. The calculated photoelectron emission probability [solid (red) curve in main

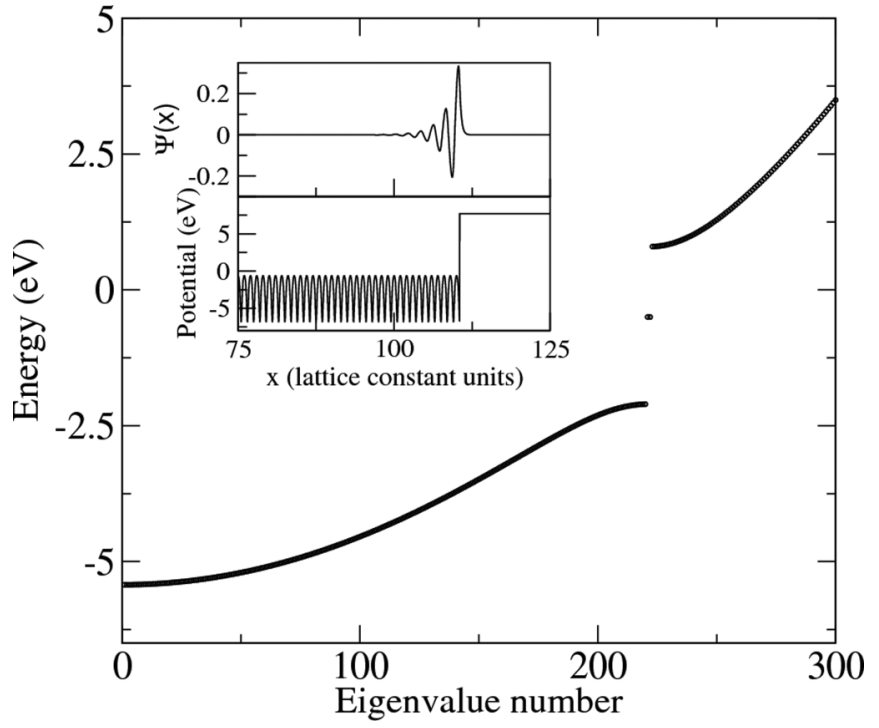

FIG. 4. Details of the field-free model Hamiltonian. The eigenvalues show the presence of a surface state within the gap, as in real $\mathrm{Au}(111)$. The Fermi level was taken as the 0 of the energy scale. Inset: (Bottom) Periodic potential modeling the linear chain of atoms and (top) wave function of the symmetric surface state.

Fig. 2] compares well with the PE intensity measured at normal emission (open circles). The best agreement is obtained for a theoretical laser intensity of $400 \mathrm{GW} / \mathrm{cm}^{2}$. This value is moderately higher than the experimental one, but the agreement is satisfactory if we consider the simplicity of the model. Our calculation further shows that one third of excited electrons actually absorbed more than one photon. The majority of these electrons remain inside the solid, with energies below the vacuum level at the end of the pulse. The relative peak intensity shown in Fig. 2 is mildly dependent on the details of the potential, but the following features are essential. First, the presence of the periodic modulation is crucial, allowing excited electrons to exchange crystal momentum with the lattice while absorbing further photons. The calculated absorption probability would indeed decrease by several orders of magnitude within a flat potential well of the same depth [39]. Second, the presence of the intermediate states for one-photon absorption processes appears to be fundamental for reproducing the experimental results, as we could check by comparing the results of simulations with 1D potentials tuned to obtain specific band structures. This suggests that intermediate states between the surface state and the valence band edge could play a role in the multiphoton PE process.

On the real surface, two mechanisms can be envisaged to allow the absorption of multiple low-energy photons passing through real instead of virtual states: indirect transitions (such as phonon-assisted ones) and the presence of a surface superstructure (namely, the $22 \times \sqrt{3}$ reconstruction). In the latter case, the folding of the $(1 \times 1) \mathrm{BZ}$ inside the much smaller $(22 \times \sqrt{3})$ one can also be thought of from the point of view of a "relaxing" of the $\mathbf{k}_{\|}$conservation in the $(1 \times 1)$ surface BZ, by allowing Umklapp processes corresponding to a change of $\mathbf{k}_{\|}$by a vector of the (denser) reciprocal lattice 


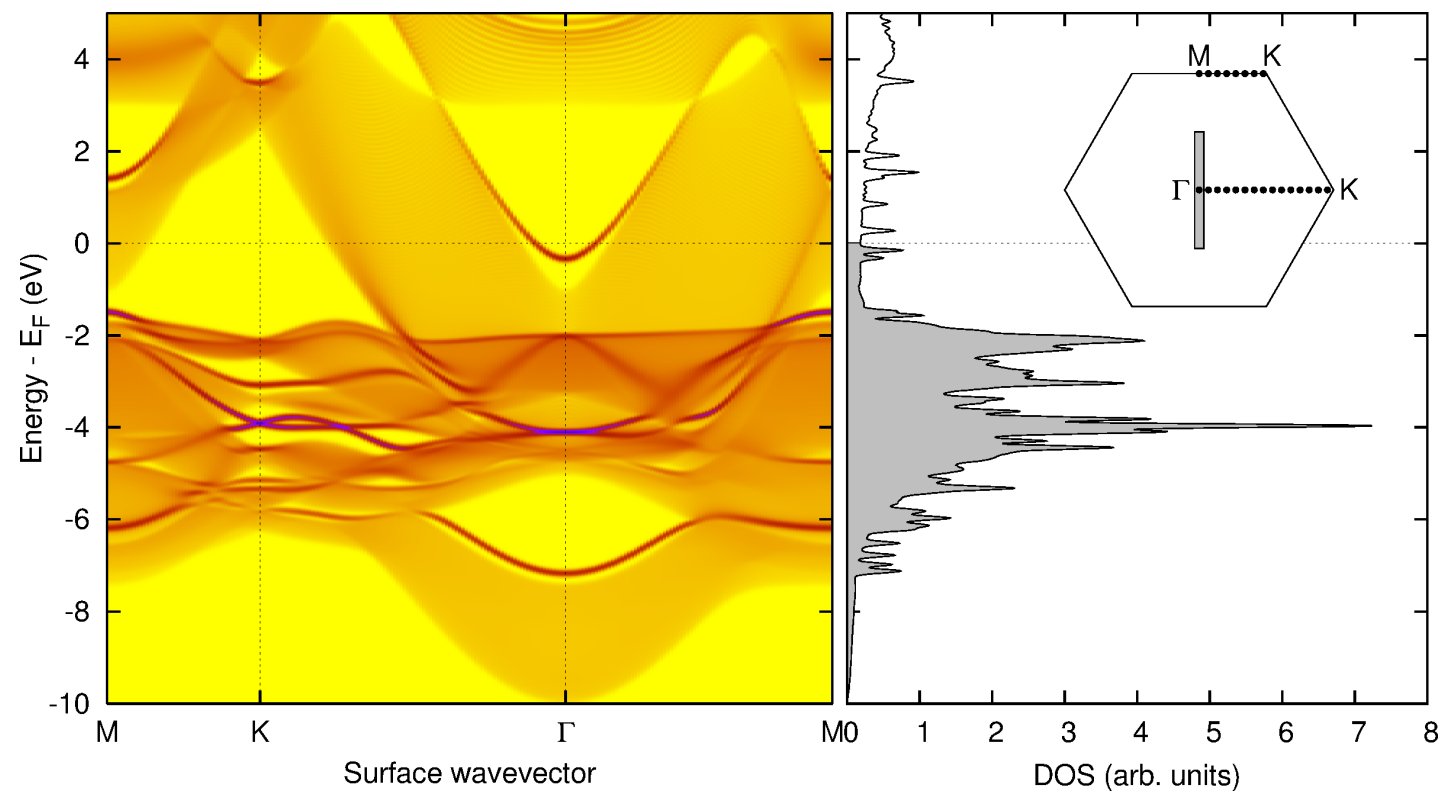

FIG. 5. (Color online) Left: Computed surface band structure on the first layer of a Au(111)- $(1 \times 1)$ slab. Right: Density of states $($ DOS $)$ at the $\Gamma$ point projected on the surface Au atom for the $22 \times \sqrt{3}$ surface reconstruction, summing contributions from $\mathbf{k}_{\|}$points which fold into the BZ center (represented by filled circles in the inset). Inset: BZ of the pristine (hexagon) and reconstructed (thin rectangle) Au(111) surface.

of the reconstructed periodicity. The density functional theory surface band structure of unreconstructed $\mathrm{Au}(111)$ is depicted in the left panel in Fig. 5, where the well-known band gap and Shockley surface state at the $\Gamma$ point are evident, so that no empty state within the $800-\mathrm{nm}$ photon energy is accessible above the surface state. States away from the $\Gamma$ point may, however, be folded in the center of the BZ as a consequence of the $22 \times \sqrt{3}$ surface reconstruction $[45,46]$. In particular, the BZ of the pristine and of the reconstructed surface are shown in the right panel in Fig. 5 as the hexagon and the thin shaded rectangle, respectively. All $\mathbf{k}_{\|}$marked by the filled circles (plus those equivalent by symmetry) fold into the $\Gamma$ point of the reconstructed BZ (including points close to $\Gamma$ and $M$, where surface states exist). The density of states (DOS) at the reconstructed $\Gamma$ point thus includes all contributions from these points and the resulting DOS projected on the surface Au atom is shown in the right panel in Fig. 5. At variance with the unreconstructed case, the DOS now displays several structures in the proximity of the Fermi level, which are mostly due to the dispersion of the surface states from $\Gamma$ and $\mathrm{M}$. There is also some structureless background due to the presence of bulk bands which extend to the surface.

\section{CONCLUSIONS}

In conclusion, we have presented the first experimental evidence of angular resolved multiphoton PE processes with four, five, six, and seven photons. A high photoelectron yield is measured with an intermediate photon energy density $\left(120 \mathrm{GW} / \mathrm{cm}^{2}\right.$ in $50-\mathrm{fs}$ pulses with a $282-\mathrm{KHz}$ repetition rate) using an 800-nm laser. Primary multiphoton electrons are generated in the first atomic layers at the surface and electrons are mainly extracted from the surface state. The measured direct PE intensity well reproduces the $E\left(\mathbf{k}_{\|}\right)$ intensity distribution expected for the electrons populating the $\mathrm{Au}(111)$ surface state with a small effective mass. Excited electrons are observed above the Fermi level with the same $E\left(\mathbf{k}_{\|}\right)$distribution observed in ARPES experiments performed with synchrotron radiation excitation. All these spectroscopic features can be easily identified, because transitions from bulk states and $d$ bands are not allowed at that photon energy in that direction. The relative intensity of the surface electronic structure replicas observed in the multiphoton ARPES experiments are well reproduced by a theoretical one-electron model based on the time-dependent Schrödinger equation for one electron in a periodic potential coupled to the laser radiation in the strong-field regime.

Based on the observed large electron yield-given by electrons which have absorbed at least four photonsassociated with the theoretical predictions of the multiphoton absorption model, we conclude that, within a single laser pulse, a significant transient population of excited states above $E_{F}+h v$ occurs. Such a high density of multiexcited electrons is expected to play an important role in a wide class of experiments performed under pulsed laser irradiation at intermediate power densities.

\section{ACKNOWLEDGMENTS}

The research described here was supported by Triangle de la physique Contract No. 2010-005T-FEMTOMAG, and we thank the European Theoretical Spectroscopy Facility (ETSF) [47] for theoretical support through User Project No. 456. 
[1] S. I. Anisimov, V. A. Benderskii, and G. Farkas, Sov. Phys. Usp. 122, 185 (1977).

[2] W. S. Fann, R. Storz, and J. Bokor, Phys. Rev. B 44, 10980(R) (1991).

[3] Gy. Farkas and Cs. Tóth, Phys. Rev. A 41, 4123(R) (1990).

[4] Gy. Farkas, Cs. Tóth, S. D. Moustaizis, N. A. Papadogiannis, and C. Fotakis, Phys. Rev. A 46, R3605(R) (1992).

[5] M. Aeschlimann, C. A. Schmuttenmaer, H. E. Elsayed Ali, R. J. D. Miller, J. Cao, Y. Gao, and D. A. Mantell, J. Chem. Phys. 102, 8606 (1995).

[6] F. Banfi, C. Giannetti, G. Ferrini, G. Galimberti, S. Pagliara, D. Fausti, and F. Parmigiani, Phys. Rev. Lett. 94, 037601 (2005).

[7] G. Saathoff, L. Miaja-Avila, M. Aeschlimann, M. M. Murnane, and H. C. Kapteyn, Phys. Rev. A 77, 022903 (2008).

[8] M. Schenk, M. Krüger, and P. Hommelhoff, Phys. Rev. Lett. 105, 257601 (2010)

[9] D. J. Park, B. Piglosiewicz, S. Schmidt, H. Kollmann, M. Mascheck, P. Gro, and C. Lienau, Ann. Physik 525, 135 (2013).

[10] F. Bisio, A. Winkelmann, C. T. Chiang, H. Petek, and J. Kirschner, J. Phys.: Condens. Matter 23, 485002 (2011).

[11] F. Bisio, M. Nývlt, J. Franta, H. Petek, and J. Kirschner, Phys. Rev. Lett. 96, 087601 (2006).

[12] L. V. Keldysh, Sov. Phys. JETP 20, 1307 (1965).

[13] G. Herink, D. R. Solli, M. Gulde, and C. Ropers, Nature 483, 190 (2012).

[14] A. Apolonski, P. Dombi, G. G. Paulus, M. Kakehata, R. Holzwarth, Th. Udem, Ch. Lemell, K. Torizuka, J. Burgdörfer, T. W. Hänsch, and F. Krausz, Phys. Rev. Lett. 92, 073902 (2004).

[15] S. Chelkowski, A. D. Bandrauk, and A. Apolonski, Phys. Rev. A 70, 013815 (2004).

[16] H. Yanagisawa, M. Hengsberger, D. Leuenberger, M. Klöckner, C. Hafner, T. Greber, and J. Osterwalder, Phys. Rev. Lett. 107, 087601 (2011).

[17] M. R. Bionta, B. Chalopin, J. P. Champeaux, S. Faure, A. Masseboeuf, P. Moretto-Capelle, and B. Chatel, J. Mod. Opt. 61, 833 (2013).

[18] E. Beaurepaire, J.-C. Merle, A. Daunois, and J.-Y. Bigot, Phys. Rev. Lett. 76, 4250 (1996).

[19] C. D. Stanciu, F. Hansteen, A. V. Kimel, A. Kirilyuk, A. Tsukamoto, A. Itoh, and Th. Rasing, Phys. Rev. Lett. 99, 047601 (2007).

[20] A. Kirilyuk, A. V. Kimel, and T. Rasing, Rev. Mod. Phys. 82, 2731 (2010).

[21] S. Mangin, M. Gottwald, C.-H. Lambert, D. Steil, V. Uhl, L. Pang, M. Hehn, S. Alebrand, M. Cinchetti, G. Malinowski et al., Nat. Mater. 13, 286 (2014).

[22] G. Malinowski, F. Dalla Longa, J. H. H. Rietjens, P. V. Paluskar, R. Huijink, H. J. M. Swagten, and B. Koopmans, Nat. Phys. 4, 855 (2008).
[23] M. Battiato, K. Carva, and P. Oppeneer, Phys. Rev. Lett. 105, 027203 (2010).

[24] D. Rudolf, C. La-o-Vorakiat, M. Battiato, R. Adam, J. M. Shaw, E. Turgut, P. Maldonado, S. Mathias, P. Grychtol, H. T. Nembach et al., Nat Commun 3, 1037 (2012).

[25] T. Wang, D. Zhu, B. Wu, C. Graves, S. Schaffert, T. Rander, L. Müller, B. Vodungbo, C. Baumier, D. P. Bernstein et al., Phys. Rev. Lett. 108, 267403 (2012).

[26] E. Turgut, C. La-o-vorakiat, J. M. Shaw, P. Grychtol, H. T. Nembach, D. Rudolf, R. Adam, M. Aeschlimann, C. M. Schneider, T. J. Silva et al., Phys. Rev. Lett. 110, 197201 (2013).

[27] A. J. Schellekens, W. Verhoeven, T. N. Vader, and B. Koopmans, Appl. Phys. Lett. 102, 252408 (2013).

[28] J. Mendil, P. Nieves, O. Chubykalo-Fesenko, J. Walowski, T. Santos, S. Pisana, and M. Mnzenberg, Sci. Rep. 4, 3980 (2014).

[29] F. Polack, M. G. Silly, C. Chauvet, B. Lagarde, N. Bergeard, M. Izquierdo, O. Chubar, D. Krizmancic, M. Ribbens, J. P. Duval et al., AIP Conf. Proc. 1234, 185 (2010).

[30] Ph. Martin, J. Phys. B: Atom. Mol. Opt. Phys. 29, L635 (1996).

[31] Ph. Martin, S. Vivirito, and G. Petite, J. Phys. B: Atom. Mol. Opt. Phys. 33, 767 (2000).

[32] M. D Feit, J. A Fleck, and A. Steiger, J. Comput. Phys. 47, 412 (1982).

[33] K. J. Schafer and K. C. Kulander, Phys. Rev. A 42, 5794(R) (1990).

[34] J. P. Perdew, K. Burke, and M. Ernzerhof, Phys. Rev. Lett. 77, 3865 (1996).

[35] P. Giannozzi, S. Baroni, N. Bonini, M. Calandra, R. Car, C. Cavazzoni, D. Ceresoli, G. L Chiarotti, M. Cococcioni, I. Dabo et al., J. Phys.: Condens. Matter 21, 395502 (2009).

[36] F. Reinert, G. Nicolay, S. Schmidt, D. Ehm, and S. Hüfner, Phys. Rev. B 63, 115415 (2001)

[37] D. Woodruff, W. A. Royer, and N. V. Smith, Phys. Rev. B 34, 764 (1986).

[38] F. H. M. Faisal and J. Z. Kamiski, Phys. Rev. A 58, R19 (1998).

[39] S. V. Yalunin, M. Gulde, and C. Ropers, Phys. Rev. B 84, 195426 (2011).

[40] V. Apalkov and M. I. Stockman, Phys. Rev. B 88, 245438 (2013).

[41] G. Banfi, G. Ferrini, M. Peloi, and F. Parmigiani, Phys. Rev. B 67, 035428 (2003).

[42] P. H. Bucksbaum, R. R. Freeman, M. Bashkansky, and T. J. McIlrath, J. Opt. Soc. Am. B 4, 760 (1987).

[43] J. Javanainen, J. H. Eberly, and Q. Su, Phys. Rev. A 38, 3430 (1988).

[44] Q. Su and J. H. Eberly, Phys. Rev. A 44, 5997 (1991).

[45] F. Reinert and G. Nicolay, Appl. Phys. A: Mater. Sci. Process. 78, 817 (2004).

[46] Y. Wang, N. S. Hush, and J. R. Reimers, Phys. Rev. B 75, 233416 (2007).

[47] A. Matsuura, N. Thrupp, X. Gonze, Y. Pouillon, G. Bruant, and G. Onida, Comput. Sci. Eng. 14, 22 (2012). 